12

\title{
Токовые характеристики сильноточной электронной пушки с многоканальным инициированием взрывной эмиссии пробоем по поверхности диэлектрика
}

\author{
(С) В.И. Петров, П.П. Кизириди, Г.Е. Озур \\ Институт сильноточной электроники СО РАН, \\ 634055 Томск, Россия \\ e-mail: ozur@Ive.hcei.tsc.ru
}

Поступило в Редакцию 26 марта 2021 г.

В окончательной редакции 20 мая 2021 г.

Принято к публикации 6 июня 2021 г.

\begin{abstract}
Исследованы токовые характеристики плазмонаполненной сильноточной электронной пушки при различных значениях ускоряющего напряжения и концентрации плазмы, предварительно заполняющей пространство между катодом и коллектором. Многоканальное инициирование взрывной эмиссии осуществляется с помощью параллельно срабатывающих, резистивно-развязанных дуговых источников плазмы, электроды и трубчатые керамические изоляторы которых встроены во взрывоэмиссионный катод. Продемонстрирована высокая эмиссионная способность (средняя по площади плотность тока) данного катодного узла, превосходящая в 1.4-2.4 раза аналогичную величину для случая традиционной пушки с плазменным анодом и многопроволочным медным взрывоэмиссионным катодом. Подтверждена возможность работы данной сильноточной электронной пушки при амплитуде ускоряющего напряжения вплоть до $30 \mathrm{kV}$.
\end{abstract}

Ключевые слова: сильноточные электронные пучки, взрывная эмиссия, катодный узел, многоканальное инициирование, пробой по поверхности диэлектрика.

DOI: 10.21883/JTF.2021.11.51541.80-21

\section{Введение}

Развитие физики и техники генерирования низкоэнергетических (10-30 keV) сильноточных электронных пучков (НСЭП) является актуальной задачей с точки зрения их использования для модификации поверхностных слоев металлических материалов [1-7]. Формирование таких пучков осуществляется, как правило, в пушках с плазменным анодом и взрывоэмиссионным катодом [4]. Наиболее актуальными задачами остаются повышение стабильности параметров НСЭП от импульса к импульсу и обеспечение однородности распределения плотности энергии по сечению пучка. Решение этих задач существенно зависит от стабильности работы взрывоэмиссионного катода электронной пушки.

Заметным шагом в деле улучшения работы сильноточной электронной пушки стал предложенный нами метод инициирования взрывной эмиссии путем встраивания в дисковый катод резистивно развязанных дуговых источников плазмы [8]. Отличительной чертой данного метода является то, что электропитание как дуговых источников плазмы, так и ускоряющего зазора электронной пушки осуществляется от одного генератора высоковольтных импульсов. Первые же эксперименты с новым катодным узлом показали улучшенную стабильность его работы по сравнению с наиболее часто используемым многопроволочным медным катодом (изготовляемым обычно из оплетки радиочастотного кабеля) и повышенную (в 1.5-1.7 раза) среднюю плотность эмиссионного тока.
Перспективность данного катодного узла обусловлена также возможностью его использования в режиме вакуумного или газонаполненного диода, благодаря независимости инициирования взрывной эмиссии от ускоряющего зазора и от его наполнения. В настоящей работе, являющейся продолжением работы [8], представлены полученные после опубликования последней результаты исследования токовых характеристик плазмонаполненной сильноточной электронной пушки.

\section{1. Методика эксперимента}

В экспериментах использовалась та же установка [8], схема которой приведена на рис. 1. Катодный узел включает в себя взрывоэмиссионный катод 1 в виде перфорированного медного диска, в отверстия которого заподлицо вставлены керамические трубки 2 и медные электроды 3. Электроды (всего 69 штук) заземлены через резисторы 4 общим сопротивлением $3 \mathrm{k} \Omega$ (три резистора номиналом $1 \mathrm{k} \Omega$ последовательно; в [8] было 2 резистора таким же номиналом). Добавление третьего резистора в цепь каждого электрода было сделано для увеличения электрической прочности катодного узла, снижавшейся до этого при напряжении выше $20 \mathrm{kV}$. Межцентровое расстояние между электродами составляет $5.5 \mathrm{~mm}$. Для усиления электрического поля и локальной фокусировки ионного потока из плазменного анода на рабочей поверхности катода нарезаны кольцевые канавки треугольного сечения. Эффективный диаметр 


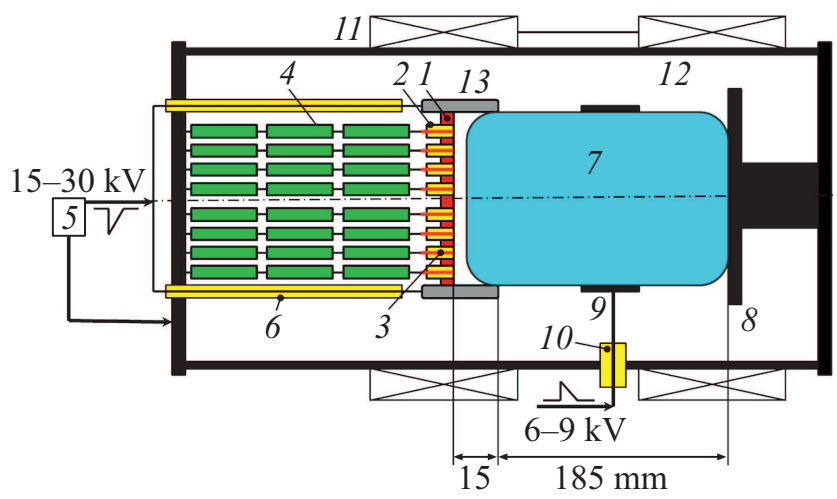

Рис. 1. Схема эксперимента (пояснения в тексте).

эмитирующей поверхности катода составлял $5 \mathrm{~cm}$, а соответствующая ему площадь $S=19.6 \mathrm{~cm}^{2}$. Для ограничения попадания электронов катодной плазмы на стенку корпуса пушки 12 установлен экранный электрод 13, электрически соединенный с катодом.

При подаче на катод импульса отрицательной полярности амплитудой $15-30 \mathrm{kV}$ от генератора высоковольтных импульсов (ГВИ) 5 через проходные изоляторы 6 происходит пробой по поверхности торцов керамических трубок. При этом возникает плазма, состоящая из ионизованных паров материалов катода (катодные пятна), керамических трубок и электродов, а также ионизованного десорбированного газа. Образование катодных пятен при протекании тока через поджигающие промежутки обеспечивается тем, что амплитуда тока (5-8 A) через каждый промежуток превышает пороговый ток дуги [9], который составляет доли-единицы А практически для всех материалов (например, для меди он равен около 2 А). Электроны, эмитируемые начальными, а также возникшими в течение импульса катодными пятнами, ускоряются в двойном слое между плазмой катодных пятен и предварительно созданным плазменным анодом 7 и транспортируются к заземленному коллектоpy 8. Рост тока обеспечивается как увеличением числа катодных пятен, так и их расширением, т. е. увеличением площади эмиссионной поверхности $[4,10,11]$.

Плазменный анод создавался с помощью сильноточного отражательного разряда (OP) при подаче импульса положительной полярности через проходной изолятор 10 на кольцевой анод 9. Ведущее магнитное поле, создаваемое секционированным соленоидом 11 , обеспечивало как зажигание и горение сильноточного ОР, так и транспортировку пучка к коллектору [4]. Величина индукции ведущего магнитного поля составляла $0.1-0.14$ Т. В ряде экспериментов использовался кольцевой анод со встроенными в него дуговыми источниками плазмы; в этом случае плазменный анод создавался уже с помощью гибридного разряда [12]. Амплитуда тока разряда составляла около 500 А для случая традиционного ОР (без дуговых источников плазмы) и около 1500 А для случая гибридного разряда. Для минимизации возможного контакта плазменного анода с экранным электродом внутренний диаметр анода был на $3 \mathrm{~mm}$ меньше внутреннего диаметра этого электрода.

Регулировка исходной концентрации анодной плазмы осуществлялась путем изменения времени задержки, $\tau_{d}$, между началом импульса тока разряда и подачей импульса ускоряющего напряжения на катод.

Управление моментами включения источников питания ведущего магнитного поля, разряда, формирующего плазменный анод, и ускоряющего напряжения осуществлялось с помощью генератора задержанных импульсов с оптической развязкой каналов. Частота посылок импульсов составляла 1 pulse/10 s.

Откачка рабочего объема пушки осуществлялась турбомолекулярным насосом до давления 0.05 mTorr; после откачки напускался рабочий газ (аргон) до давлений $0.3-0.5$ mTorr.

Регистрация импульсов ускоряющего напряжения осуществлялась с помощью активного делителя, тока катода - поясом Роговского, тока пучка - малоиндуктивным шунтом сопротивлением $0.005 \Omega$ или поясом Роговского. Сигналы с датчиков подавались на входы 4-канального широкополосного (200 MHz) цифрового осциллографа Tektronix TDS 2024. В каждом режиме производилось 10 выстрелов.

\section{2. Результаты экспериментов и обсуждение}

На рис. 2 приведена серия типичных осциллограмм импульсов, полученных при различных значениях зарядного напряжения ГВИ, $U_{c h}$. Видно, что ток пучка на коллектор слабо меняется с изменением ускоряющего напряжения. Это соответствует известному свойству квазистационарных двойных слоев в плазме: независимости плотности тока в двойном слое от приложенного напряжения [13-5].

Аналогичное поведение тока пучка в зависимости от ускоряющего напряжения проявилось и в случае формирования плазменного анода с помощью гибридного разряда (рис. 3).

Более заметное увеличение тока пучка с ростом $U_{c h}$, чем на рис. 2 , возможно связано с проявлением нестационарности двойного слоя при быстром росте ускоряющего напряжения на фронте импульса [4], либо со случайным отклонением начальной концентрации анодной плазмы.

Увеличение тока пучка наблюдалось, как и следовало ожидать, при увеличении исходной концентрации анодной плазмы, что иллюстрируется осциллограммами, приведенными на рис. 4. Видно, что с ростом времени задержки $\tau_{d}$ с 15 до $35 \mu \mathrm{s}$ среднее значение тока пучка в первой половине импульса (длительностью около $1 \mu \mathrm{s}$ ) уменьшается примерно в три раза, что соответствует результатам измерений концентрации ионов в плазмен- 


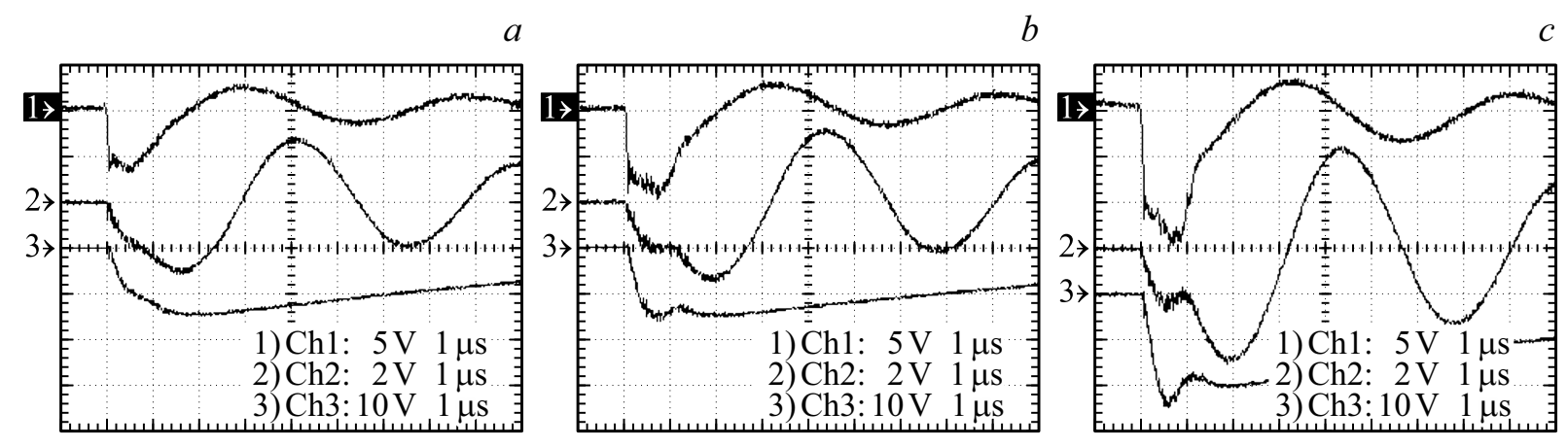

Рис. 2. Типичные осциллограммы ускоряющего напряжения (Ch1, $10 \mathrm{kV} / \mathrm{div})$, полного тока катода $(\mathrm{Ch} 2,24 \mathrm{kA} / \mathrm{div})$ и тока пучка на коллектор $(\mathrm{Ch} 3,10 \mathrm{kA} / \mathrm{div})$; горизонтальная шкала - $1 \mu \mathrm{s} / \mathrm{div}: U_{c h}=15 \mathrm{kV}(a), 20 \mathrm{kV}(b), 30 \mathrm{kV}(c)$. Давление аргона - $0.5 \mathrm{mTorr}$, $\tau_{d}=15 \mu \mathrm{s}$, кольцевой анод.

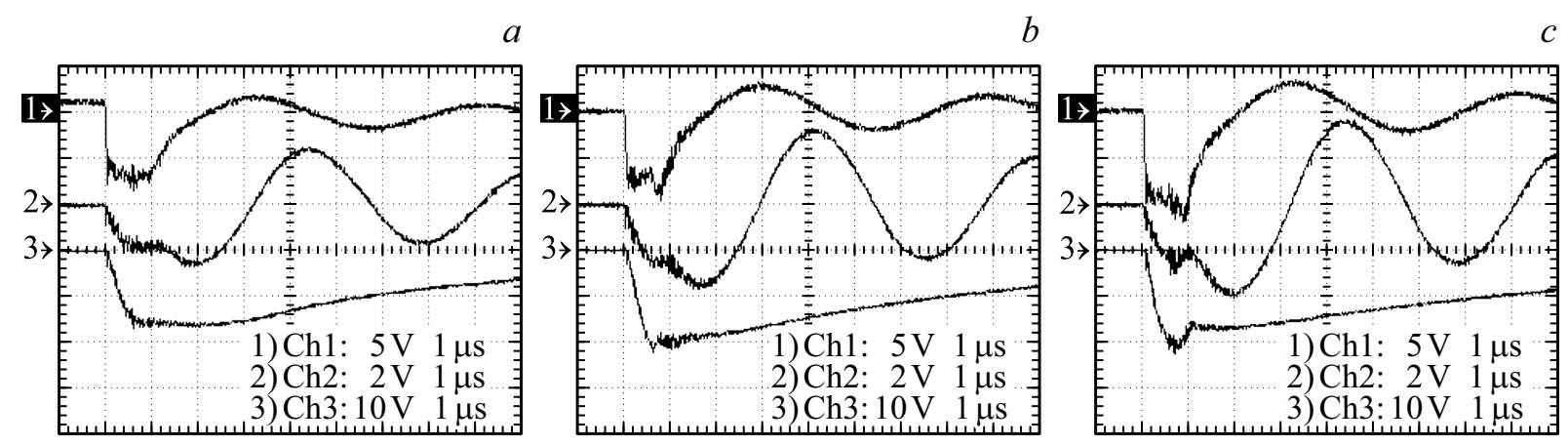

Рис. 3. Типичные осциллограммы ускоряющего напряжения (Ch1, $10 \mathrm{kV} / \mathrm{div})$, полного тока катода (Ch2, 24kA/div) и тока пучка на коллектор $(\mathrm{Ch} 3,10 \mathrm{kA} / \mathrm{div}) ;$ горизонтальная шкала $-1 \mu \mathrm{s} / \mathrm{div}: U_{c h}=15 \mathrm{kV}(a), 20 \mathrm{kV}(b), 25 \mathrm{kV}(c)$. Давление аргона - $0.5 \mathrm{mTorr}$, $\tau_{d}=20 \mu \mathrm{s}$, гибридный анод.

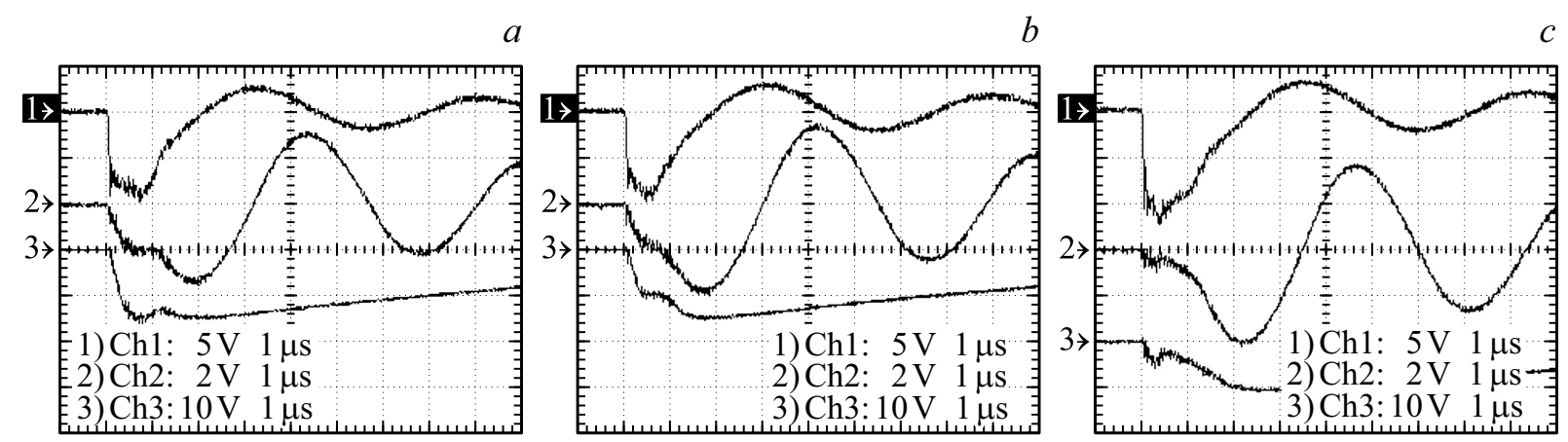

Рис. 4. Типичные осциллограммы ускоряющего напряжения (Ch1, $10 \mathrm{kV} / \mathrm{div})$, полного тока катода (Ch2, 24kA/div) и тока пучка на коллектор $(\mathrm{Ch} 3,10 \mathrm{kA} / \mathrm{div})$; горизонтальная шкала $-1 \mu \mathrm{s} / \mathrm{div}: \tau_{d}=15 \mu \mathrm{s}(a) ; \tau_{d}=25 \mu \mathrm{s}(b) ; \tau_{d}=35 \mu \mathrm{s}(c)$. Давление аргона $0.5 \mathrm{mTorr}, U_{c h}=20 \mathrm{kV}$, кольцевой анод.

ном аноде, полученным нами ранее с помощью двойных зондов (рис. 5) [16].

Следует также отметить, что при больших временах задержки $\left(\tau_{d}>35 \mu \mathrm{s}\right)$, существенно возрастала нестабильность токов катода и пучка от импульса к импульсу, что, вероятно, обусловлено низкой концентрацией анодной плазмы, а, следовательно, ухудшением условий для образования взрывоэмиссионных центров на катоде и устойчивости транспортировки пучка к коллектору.
Рост тока катода, начинающийся примерно через $1 \mu \mathrm{s}$ после начала импульса, обусловлен развитием пробоя в диоде с переходом разряда в дуговую стадию с колебательным режимом протекания тока (см. осциллограммы импульсов ускоряющего напряжения и тока катода). Пробой диода обусловлен, прежде всего, утечками тока в радиальном направлении, которые вызваны аномальной диффузией электронов, эмитированных из периферийной области катодной плазмы, поперек силовых линий ведущего магнитного поля $[4,17,18]$. Такое 
Сравнительные характеристики сильноточных электронных пушек с традиционными многопроволочными медными катодами и с новым катодным узлом

\begin{tabular}{|c|c|c|c|c|c|}
\hline Тип катода & Ток катода, kA & $\begin{array}{c}\text { Ток пучка } \\
\text { на коллектор, кА }\end{array}$ & $\begin{array}{c}\text { Эффективная площадь } \\
\text { эмиссии, } \mathrm{cm}^{2}\end{array}$ & $\begin{array}{c}\text { Плотность тока } \\
\text { катода/пучка, } \mathrm{A} / \mathrm{cm}^{2}\end{array}$ & Источник \\
\hline $\begin{array}{l}\text { Многопроволочный } \\
\text { медный }\end{array}$ & 24 & 18 & 28.3 & $848 / 636$ & {$[4]$} \\
\hline $\begin{array}{l}\text { Многопроволочный } \\
\text { медный }\end{array}$ & 30 & 29 & 56.7 & $529 / 511$ & {$[12]$} \\
\hline $\begin{array}{l}\text { Новый катодный } \\
\text { узел }\end{array}$ & 25 & 一 & 19.6 & $1276 /-$ & {$[8]$} \\
\hline $\begin{array}{l}\text { Новый катодный } \\
\text { узел }\end{array}$ & 24 & 20 & 19.6 & $1224 / 1020$ & $\begin{array}{c}\text { Настоящая } \\
\text { работа }\end{array}$ \\
\hline
\end{tabular}

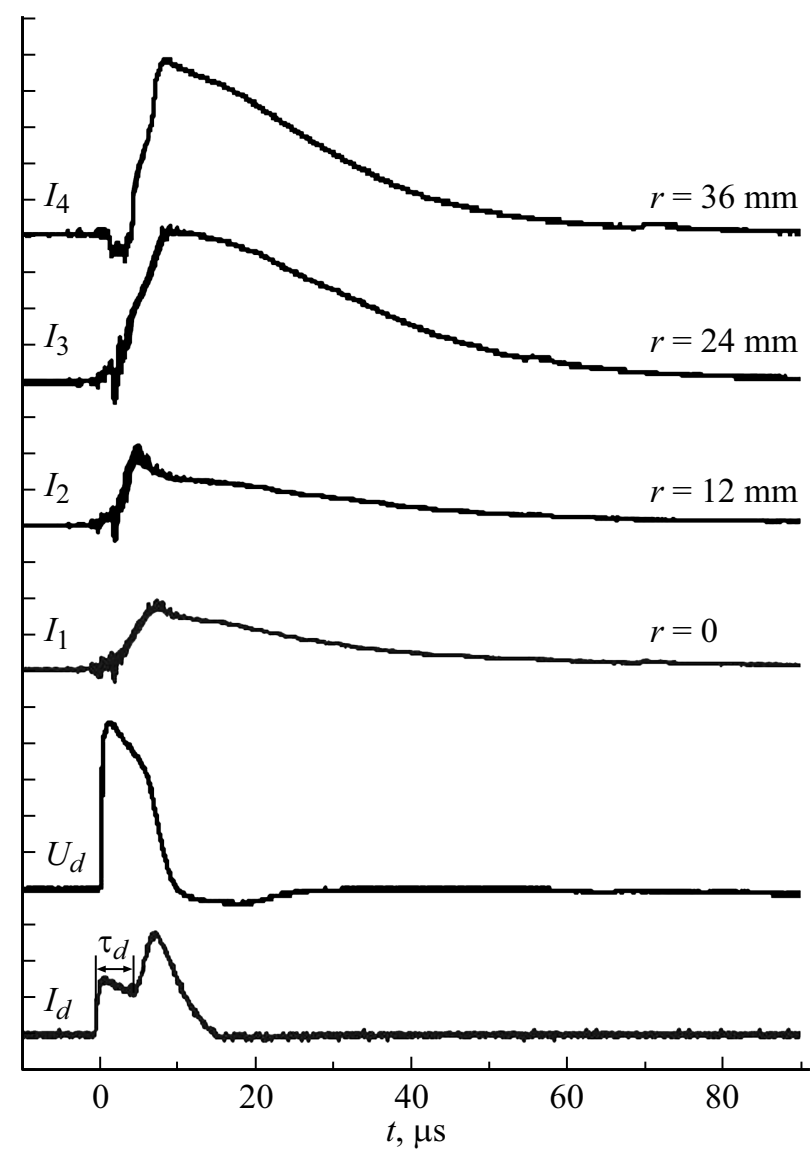

Рис. 5. Осциллограммы импульсов напряжения на аноде разряда $\left(U_{d}, 1 \mathrm{kV} / \mathrm{div}\right)$, тока разряда $\left(I_{d}, 400 \mathrm{~A} / \mathrm{div}\right)$ и зондовых токов $\left(I_{1}-I_{4}, 0.025 \mathrm{~A} / \mathrm{div}\right)$. Гибридный анод [16].

поведение сильноточного диода с плазменным анодом на основе сильноточного ОР неоднократно наблюдалось нами ранее [4]. Наличие экранного электрода 13 не может воспрепятствовать процессу пробоя, поскольку плазма взрывоэмиссионных центров довольно быстро (по нашим оценкам - за $0.3-0.4 \mu$ s при скорости катодной плазмы около $2 \mathrm{~cm} / \mu \mathrm{s}[10,11])$ выйдет на кромку этого электрода, стимулируя возникновение на нем новых взрывоэмиссионных центров за счет так называемого эффекта „подхвата“, т.е. возникновения этих центров при зарядке ионным током из плазмы (в данном случае катодной) неметаллических включений и пленок и их последующим пробоем [19,20].

Сравнение полученных в настоящей работе токовых характеристик сильноточной электронной пушки с аналогичными характеристиками, полученными ранее для пушки с традиционным многопроволочным медным катодом [4], показывает увеличенную примерно в 1.4-2.4 раза среднюю (по площади) плотность тока катода, а плотность тока пучка на коллектор - примерно в 1.6-2 раза (см. таблицу). В таблице приводятся значения тока катода и тока пучка на коллектор к моменту окончания переднего фронта импульса, т.е. через $0.3-0.5 \mu \mathrm{s}$ после начала импульса. Эти величины наиболее адекватно характеризуют эмиссионную способность катода и пропускную способность слоя. Второй максимум на осциллограмме тока катода есть ток короткого замыкания, т.е. когда пучок ускоренных электронов фактически прекратил существование; эта стадия не представляет интереса. Наблюдаемое увеличение средней плотности тока для случая катодного узла со встроенными дуговыми источниками плазмы обусловлено, на наш взгляд, не только возросшим количеством одновременно функционирующих взрывоэмиссионных центров, но и увеличенной скоростью разлета катодной плазмы, по крайней мере, в первой половине импульса. Поскольку встроенные в катод дуговые источники плазмы являются одновременно плазменными пушками, то скорость разлета этой плазмы вдоль силовых линий магнитного поля в разы выше, чем скорость разлета плазмы взрывоэмиссионного центра [4,21]. Увеличение же скорости движения эмиссионной границы катодной плазмы должно приводить, согласно [4], к увеличению пропускной способности двойного слоя, а значит, и тока пучка на коллектор.

Относительный разброс (среднеквадратичное отклонение) как амплитудных, так и средних за импульс значений тока пучка в оптимальных режимах генерирования (времена задержки $\tau_{d}=15-25 \mu$ s после начала 
импульса тока разряда) составил $4.3-7.6 \%$, что в $2-3$ раза меньше аналогичных значений для случая многопроволочного медного катода.

\section{Заключение}

1. Подтверждена высокая эмиссионная способность катодного узла со встроенными в него резистивно развязанными дуговыми источниками плазмы, которая в 1.4-2.4 раза превосходит соответствующую величину, характерную для традиционной схемы пушки с плазменным анодом и многопроволочным медным взрывоэмиссионным катодом.

2. В оптимальных режимах работы данный катодный узел обеспечивает относительный разброс тока пучка на коллектор в 2-3 раза меньший, чем в случае наиболее часто использовавшегося ранее многопроволочного медного катода (также в оптимальных режимах).

3. Добавление третьего резистора в цепь каждого дугового источника плазмы повысило электрическую прочность катодного узла, что позволило использовать импульсы ускоряющего напряжения амплитудой до $30 \mathrm{kV}$ и тем самым пропорционально повысить плотность энергии пучка.

\section{Финансирование работы}

Работа финансировалась в рамках государственного задания по программе фундаментальных научных исследований Сибирского отделения РАН „Низкотемпературная газоразрядная плазма и ее применение для решения научных и технологических задач“, проект „Низкотемпературная плазма стационарных и импульсных разрядов в газах и вакууме и ее применение в электрофизических устройствах“ № FWRM-2021-0007.

\section{Конфликт интересов}

Авторы подтверждают, что у них нет конфликта интересов.

\section{Список литературы}

[1] S.N. Meisner, E.V. Yakovlev, V.O. Semin, L.L. Meisner, V.P. Rotshtein, A.A. Neiman, F. D’yachenko. Appl. Surf. Sci., 437, 217 (2018). https://doi.org/10.1016/j.apsusc.2017.12.107

[2] Y. Uno, A. Okada, K. Uemura, P. Raharjo, T. Furukawa, K. Karato. Precis. Eng., 29, 449 (2005). DOI:10.1016/j.precisioneng.2004.12.005

[3] J.W. Murray, A.T. Clare. J. Mater. Process. Tech., 212, 2642 (2012). http://dx.doi.org/10.1016/j.jmatprotec.2012.07.018

[4] Г.Е. Озур, Д.И. Проскуровский. Физика плазмы, 44 (1), 21 (2018). DOI: 10.7868/S0367292118010146 [G.E. Ozur, D.I. Proskurovsky. Plasma Phys. Reports, 44 (1), 18 (2018). DOI: 10.1134/S1063780X18010130]
[5] L.L. Meisner, V.P. Rotshtein, V.O. Semin, S.N. Meisner, A.B. Markov, E.V. Yakovlev, F.A. D'yachenko, A.A. Neiman, E.Yu. Gudimova. Surf. Coat. Technol., 404, 12644 (2020). https://doi.org/10.1016/j.surfcoat.2020.126455

[6] J.W. Murray, P.K. Kinnell, A.H. Cannon, B. Bailey, A.T. Clare. Precis. Eng., 37, 443 (2013). http://dx.doi.org/10.1016/j.precisioneng.2012.11.007

[7] B. Gao, L. Hu, S. Li, Y. Hao, Y. Zhang, G. Tu, T. Grosdidier. Appl. Surf. Sci., 346, 147 (2015). http://dx.doi.org/10.1016/j.apsusc.2015.04.029

[8] П.П. Кизириди, Г.Е. Озур. Письма в ЖТФ, 46 (15), 47 (2020). DOI: 10.21883/JTF.2021.11.51541.80-21 [P.P. Kiziridi, G.E. Ozur, Tech. Phys. Lett., 65 (2), 297 (2020). DOI: 10.1134/S1063784220020097]

[9] И.Г. Кесаев. Катодные процессы электрической дуги (Наука, М., 1968)

[10] Г.А. Месяц, Д.И. Проскуровский. Импульсный электрический разряд в вакууме (Наука, Новосибирск, 1984)

[11] Г.А. Месяц. Эктоны в вакуумном разряде: пробой, искра, дуга (Наука, М., 2000)

[12] G.E. Ozur. Proc. of the 25th Int. Symp. on Discharges and Electrical Insulation in Vacuum (Tomsk, Russia, 2012), p. 580. DOI: 10.1109/DEIV.2012.6412586

[13] Е.И. Луценко, Н.Д. Середа, Л.М. Концевой. ЖТФ, 45 (4), 789 (1975).

[14] Е.И. Луценко, Н.Д. Середа, Л.М. Концевой. Физика плазмы, 2 (1), 72 (1976).

[15] Е.И. Луценко, Н.Д. Середа, А.Ф. Целуйко. ЖТФ, 58 (7), 1299 (1988).

[16] G.E. Ozur, P.P. Kiziridi. Proc. of the 7th International Congress on Energy Fluxes and Radiation Effects (Tomsk, Russia, 2020), p. 178. DOI: 10.1109/EFRE47760.2020.9242114

[17] М.В. Незлин. Динамика пучков в плазме (Энергоатомиздат, М., 1982)

[18] А.В. Гордеев. Физика плазмы, 32 (9), 847 (2006).

[19] Д.И. Проскуровский, В.Ф. Пучкарев. ЖТФ, 49 (12), 2611 (1979).

[20] M.A. Lutz. IEEE Trans. Plasma Sci., 2 (1), 1 (1974).

[21] W.H. Bostik. Phys. Rev. D, 104 (2), 292 (1956). 